\title{
INDUCTION DE PROTÉINES INTERVENANT DANS LA RÉPARATION DE L'ADN ALKYLE, DANS LES CELLULES IRRADIÉES
}

\author{
P. LEFEBVRE, Y. HABRAKEN et F. LAVAL
} U 247 INSERM, Institut Gustave Roussy,
94805 Villejuif

\begin{abstract}
$L^{\prime}$ induction de gènes est un phẻnomène qui a été décrit chez les bactéries (E.coli) (réponse sos, adaptation aux agents alkylants, adaptation au stress oxydatif), ainsi que dans les cellules eukaryotes. Dans les cellules de mammifères, il a été montré que l'induction de certains gènes pouvait être causée par des lésions de l'ADN ou un arrêt de la croissance. Il s'agit par exemple d'oncogènes ( c-fos, c-jun), ou de gènes codant pour la protéine kinase $c$, l'interleukine, la métallothionéine.
\end{abstract}

Nous avons recherché si des protéines intervenant dans la réparation de I'ADN pouvaient être induites dans les cellules de mammifères traitées par des agents génotoxiques, en particulier par les radiations ionisantes. Nous nous sommes plus particulièrement intéréssés à deux protéines, la N3-méthyladénine-ADN -glycosylase ( 3-meAde glycosylase) et la $0^{6}$-méthylguanine-ADN-méthyltransférase (transférase), pour les raisons suivantes. La 3-meAde glycosylase élimine la 3-méthyladénine, qui constitue un arrêt de la réplication et est donc une lésion létale formée par les drogues alkylantes. La transférase répare la $0^{6}$-méthylguanine, qui est une lésion particulièrement mutagène formée par les agents alkylants. Elle répare également les résidus $0^{6}-$ chloroêthyl guanine, qui sont des précurseurs de pontages intrabrins dans l'ADN (lésions lêtales) et qui sont formés par les chloroéthylnitrosourées utilisées en chimiothérapie anticancéreuse. Il faut rappeler que la transférase est une protéine "suicide" qui n'est pas régénérée après avoir été alkylée et le nombre de molécules de transférase par cellule est déterminant pour la sensibilité de cette cellule vis à vis de l'effet toxique et mutagène des drogues alkylantes.

Nos résultats ont montré que l'activité de la transférase ( et à un degré moindre celle de la 3-meAde glycosylase) était augmentée dans des cellules dérivées d'un hépatome de rat traitées par les radiations ionisantes. Cette augmentation est proportionnelle à la dose de radiations ( 0 à 5 Gy ). Elle est maximale 48 heures après l'irradiation, puis l'activité diminue. Cependant si les cellules recoivent 
des irradiation répétées, l'activité de ces deux protéines est augmentée aussi longtemps que les cellules sont soumises aux radiations (Lefebvre et Laval, 1986).

Cette induction semble liée à la formation de coupures I'ADN cellulaire. En effet, d'autres traitements produisant directement ou indirectement des coupures ( irradiation UV, drogues, enzymes de restriction ) induisent ces protéines, alors que dans les cellules traitées par différents composés modifiant le métabolisme cellulaire ( hydroxyurée, agents découplants, par exemple ) mais n'interagissant pas directement avec I'ADN, ce phénomène n'est pas observé (Laval, 1990).

Pour êtudier le mécanisme conduisant à cette induction, nous avons cloné les gènes codant pour la 3-meAde glycosylase (O'Connor et Laval, 1990) et pour la transférase (Rahden-Staron et Laval, 1991). En utilisant ces cDNAs comme sondes, nous avons pu montrer que l'induction était due à une augmentation de la transcription et non à une amplification de I'ADN (Laval, 1991).

Une étude similaire a montré que I'induction de ces deux protéines se produisait également dans des cellules tumorales humaines ( cellules dérivëes d'un hépatome et cellules dérivées d'un glioblastome, par exemple) ( Lefebvre et Laval, sous presse).

L'induction de ces protéines par les radiations a un effet biologique. En effet, nous avons montré, en traitant les cellules par un agent alkylant radioactif, que l'élimination de la 3-méthyladénine et de la $0^{6}$ méthylguanine était plus rapide et plus efficace dans les cellules irradiées, comparativement aux cellules témoins. Par ailleurs, les cellules irradiées deviennent plus résistantes vis à vis de l'effet létal des chloroêthyl nitrosourées utilisées en clinique ( $B C N U$ ) ( Habraken et Laval, 1991 ).

Etant donné que ce phénomène a été observé dans des cellules tumorales, l'induction de ces protéines de réparation dans les cellules irradiées pourrait jouer un rôle important lors de traitements utilisant radio et chimiothérapie.

\section{Réferences}

Lefebvre, P. and Laval,F. 1986. Enhancement of $0^{6}-$ methylguanine-DNA-methyltransferase activity induced by various treatments in mammalian cells. Cancer Res. 46, 57015705 
Laval,F. 1990. Induction of proteins involved in the repair of alkylated bases in mammalian cells by DNA-damaging agents. Mut. Res. 233, 211-218.

o'Connor, T.R. and Laval,F. 1990. Isolation and structure of a cDNA expressing a mammalian 3-methyladenine-DNAglycosylase. EMBO J. 9, 3337-3342.

Rahden-Staron, $I$. and Laval,F. 1991. CDNA cloning of the rat $0^{6}$-methylguanine-DNA-methyltransferase. Biochem. Biophys. Res. Comm. 177, 597-602.

Laval,F. 1991. Increase of $0^{6}$-methylguanine-DNAmethyltransferase and N3-methyladenine-DNA-glycosylase RNA transcripts in rat hepatoma cells treated with DNA-damaging agents. Biochem. Biophys. Res. Comm. 176, 1086-1092.

Lefebvre, $P$. and Laval, F. Induction of $0^{6}$-methylguanine-DNAmethyltransferase and N3-methyladenine-DNA-glycosylase in human cells exposed to DNA-damaging agents. DNA and cell Biol. sous presse.

Habraken, Y. and Laval,F. 1991. Enhancement of 1,3-bis(2chloroethyl)-1-nitrosourea resistance by -irradiation or drug pretreatment in rat hepatoma cells. Cancer Res. 52, $1217-1220$. 